\title{
A Tool for the Treatment of Gastrointestinal Tumors: Micro- Ecological Immunotherapy
}

\author{
Zhu Jiang Dai ${ }^{1}$, Qi Wu ${ }^{1 \#}$, Jing Qiu Zhang ${ }^{2 \#}$, Jia Ming Xu², Han Jian Zhu ${ }^{2}$, Jin $\mathrm{Gao}^{2}$, Zhuang Zhuang \\ $\mathrm{Liu}^{2}$, Dong Tang ${ }^{2}$, Dao Rong Wang ${ }^{2}$
}

${ }^{1}$ Clinical Medical College, Yangzhou University, Yangzhou, Jiangsu 225000, China; ${ }^{2}$ Department of General Surgery, Institute of General Surgery, Clinical Medical College, Yangzhou University, Northern Jiangsu People's Hospital, Yangzhou, Jiangsu 225000, China

\begin{abstract}
It has been proposed that the intestinal microbiota and gastrointestinal tumors are interdependent. Changes in the microbiota can cause dysfunction of the gastrointestinal tract, thereby promoting carcinogenic changes, leading to the occurrence of gastrointestinal tumors. Recent studies on intestinal microbiota have opened up a new area in intestinal micro-ecological immunotherapy. The intestinal microbiota is a double-edged sword. Gut microbes participate in carcinogenesis, but can also be used for immunotherapy. The intestinal microbiota is also regulated by the daily diet. Intestinal micro-ecological immunotherapy combines intestinal immune nutrition and intestinal ecological nutrition to make full use of the intestinal microbiota to strengthen nutritional support. Micro-ecological immunotherapy enhances the body's immune function by providing energy, improving the functional state of tissues and organs, protecting the intestinal mucosal barrier and maintaining normal intestinal microbiota balance. This involves, to some extent, PD-1 and PD-L1. The microbiota is beneficial to improve the clinical efficacy of conventional anti-cancer therapy and to reduce the incidence of complications. At the same time, micro-ecological immunotherapy is itself active and effective in the perioperative treatment of tumors, which is of great significance for the prognosis of the patient. Gastrointestinal tumors are increasingly linked to intestinal microbiota, and various microbiota-related technologies and drugs have been developed. In the future, the intestinal microbiota may represent a screening marker for gastrointestinal tumors. In addition, clinicians may be able to prevent and treat cancers by changing the gene expression levels of certain microbiota, or by regulating the types of microbes present.
\end{abstract}

Key words: Micro-ecological immunotherapy; Immune nutrition; Ecological nutrition; Gastrointestinal tumors; Microbiota

\section{Introduction}

There are abundant microbial communities in the gastrointestinal environment, which are closely related to the physiological and pathological state of the gastrointestinal tract. In recent years, the occurrence of gastrointestinal tumors has been increasingly linked to the activity of the intestinal microbiota. Disorders of the intestinal microbiota can lead to abnormalities in the function of the gastrointestinal tract, thereby promoting the occurrence of gastrointestinal tumors [1]. The intestinal microbiota is also regulated by the daily diet. Importantly, the intestinal microbiota is often a double-edged sword, which can both promote and eradicate gastrointestinal tumors, depending on

\#Zhu Jiang, Qi Wu and Jing Qiu Zhang contributed equally to this work.

Corresponding author: Dong Tang, $\mathrm{MD}, \mathrm{PhD}$, Department of General Surgery, Institute of General Surgery, Northern Jiangsu Province Hospital, Clinical Medical College, Yangzhou University, Yangzhou 225001, China; Tel: +86 1892783 556; +86 05148737 3395; Email: 83392785@qq.com; Dao Rong Wang, MD, PhD, Department of General Surgery, Institute of General Surgery, Northern Jiangsu Province Hospital, Clinical Medical College, Yangzhou University, Yangzhou 225001, China; Tel: +86 1390525 2590; 05148737 3395; Email: daorong666@sina.com the types of microbes present and other conditions present [2]. Recent research on intestinal microbiota has revealed that micro-ecological immunotherapy may represent a novel approach to preventing and/or treating gastrointestinal cancers. Micro-ecological immunotherapy employs a combination of intestinal immune nutrition and intestinal ecological nutrition $[3,4]$. This article will elaborate on the effects of intestinal micro-ecological immunotherapy, including ways to provide energy, improve the functional states of tissues and organs, improve the body's immune function, and protect the gastrointestinal mucosal barrier by providing an optimal balance in microbes [5]. We also discuss the effects of micro-ecological immunotherapy on perioperative cancer treatment, and the responses to radiotherapy and chemotherapy, as well as the local microenvironment [6]. Gastrointestinal microbes are also expected to be useful as markers to screen for gastrointestinal tumors, and microbes are becoming a new focus for gastrointestinal cancer prevention and treatment.

\section{Epidemiology of Gastrointestinal Tumors}

Despite significant progress in the diagnosis and treatment of gastrointestinal tumors, the incidence and mortality rates of gastrointestinal tumors are still high due to 
factors such as heredity, lifestyle, mental state and infections. At present, the incidence and mortality of gastrointestinal tumors ranks at the forefront of all types of tumors in both men and women in China [7]. These tumors reduce the quality of life of patients and cause a serious burden on the social economy. At the same time, the prognosis of gastrointestinal tumors remains a major problem that plagues clinicians and researchers [8]. Among the various pathogenic factors associated with gastrointestinal tumors, disorders of the gastrointestinal microbiota and infectious factors are being increasingly found to play a decisive role in the occurrence, development and progression of tumors.

\section{Gastrointestinal Microbiota and Tumorigenesis}

The stomach has long been considered sterile, but it actually contains a variety of microbes. One such microbe is Helicobacter pylori (H. pylori). In many parts of the world, the infection rate of $H$. pylori correlates with the rate of gastric cancer. H. pylori can cause pathological changes in the host stomach, including chronic gastritis, atrophy, intestinal metaplasia and gastric cancer [9]. The bacterium secretes vacuolar cytotoxins such as $\mathrm{CagL}$ and VacA, which interact with mucosal epithelial cells, leading to changes in signaling pathways and cell polarity, which ultimately lead to the development of gastric cancer [10]. These oncoproteins activate different pathways, such as Csk/Crk/ SHP-2 (through a phosphorylation-dependent pathway), leading to cytoskeletal reorganization and cell death [11]. CagA also activates Crb2/c-Met via a phosphorylationindependent pathway, resulting in pro-inflammatory and mitogenic gene expression, which also leads to cancer formation [12]. VacA has immunomodulatory and inhibitory effects on various mammalian immune cells, ensuring the persistence of $\mathrm{H}$. pylori in the host. VacA can inhibit the activation and proliferation of both $\mathrm{T}$ cells and $B$ cells and interferes with the antigen expression of $B$ cells [13]. VacA prevents normal antigen processing, phagocytic expression, intracellular killing and cytokine production. Animal experiments also revealed that VacA can cause damage to the gastric mucosa, increase the recruitment of inflammatory cells, and induce cancer [14].

There are thousands of microbes in the human intestine, which form a symbiotic system with the intestinal cells to maintain the intestinal balance. However, some intestinal microbes are pathogenic or carcinogenic, with some exerting these effects when the intestinal environment is disturbed. For example, Enterococcus faecalis infection causes colitis and the expression of TGF- $\beta$, which activates the Smad4 signaling pathway. It also produces extracellular superoxide, participates in bystander effects, causes DNA damage, and thus induces cancer [15]. The B2 strain of $E$. coli produces cell necrosis factor, which inhibits mismatch repair. When these bacteria also produce pathogenic pks, the chromosome can become unstable and cancers can occur [16]. Bacteroides fragilis releases an enterotoxin (BFT), activates the STAT3 signaling pathway, induces the production of Th17 and interleukin-17, and promotes colon cancer formation [17]. The BFT can rapidly induce the expression of SMO, promoting the production of ROS, and destroying the DNA of intestinal epithelial cells [18]. At the same time, BFT is able to amplify signaling via the Wnt and NF- $\mathrm{KB}$ signaling pathways, and leads to the release of pro-inflammatory substances. Fusobacterium nucleatum stimulates FadA, activates wnt/ $\beta$-catenin/TLR4 signaling and upregulates oncogene expression [17], and Fusobacterium nucleatum can inhibit the activity of natural killer cells in the tumor microenvironment, leading to the occurrence of colorectal cancer [19].

\section{Relationship between Changes in Intestinal Microbiota and Intestinal Nutrition}

Undoubtedly, changes in the intestinal microbiota are also closely related to intestinal nutrients. The most direct source of intestinal nutrition is the daily diet. Carbohydrates are divided into digestible carbohydrates and non-digestible carbohydrates. Digestible carbohydrates are enzymatically degraded in the small intestine, including starches and sugars such as glucose, fructose, sucrose and lactose. The consumption of high levels of glucose, fructose and sucrose increases the relative abundance of bifidobacteria,

Table 1 Relationship between changes in the intestinal microbiota and intestinal nutrition.

\begin{tabular}{|c|c|c|c|c|}
\hline \multirow[t]{2}{*}{ Intestinal } & \multirow{2}{*}{$\begin{array}{l}\text { Nutrition } \\
\text { composition }\end{array}$} & \multirow[t]{2}{*}{ Example dietary sources } & \multicolumn{2}{|c|}{ Changes in the intestinal microbiota } \\
\hline & & & Increase & Decrease \\
\hline \multirow[t]{2}{*}{ Carbohydrates } & $\begin{array}{c}\text { Digestible } \\
\text { carbohydrate }\end{array}$ & $\begin{array}{c}\text { Starch, sugar } \\
\text { (glucose, fructose, lactose) }\end{array}$ & Bifidobacteria & $\begin{array}{l}\text { Bacteroides spp., } \\
\text { Clostridium spp. }\end{array}$ \\
\hline & $\begin{array}{l}\text { Non-digestible } \\
\text { carbohydrate }\end{array}$ & $\begin{array}{l}\text { Fiber (whole wheat, wheat } \\
\text { bran, resistant starch) }\end{array}$ & $\begin{array}{l}\text { Bifidobacteria Lactobacilli, Rumenococci, E. rec- } \\
\text { tale, Roseburia spp. }\end{array}$ & \\
\hline \multirow[t]{2}{*}{ Fats } & Saturated fat & Fat, butter & $\begin{array}{l}\text { Clostridium spp., Bacteroides spp., Faecalibacte- } \\
\text { ria, }\end{array}$ & Lactobacilli \\
\hline & Unsaturated fat & Fish & Bifidobacteria, Streptococci & \\
\hline \multirow[t]{2}{*}{ Proteins } & Animal protein & Dairy products & Bacteroides spp., Alistipes spp., Clostridium spp. & Bifidobacteria \\
\hline & Plant protein & Bean products & Bifidobacteria, Lactobacilli & $\begin{array}{l}\text { Bacteroides spp., } \\
\text { Clostridium spp. }\end{array}$ \\
\hline
\end{tabular}


while the abundance of Bacteroides species is relatively reduced [20]. The addition of lactose to the diet replicates the same bacterial changes and also reduces the abundance Clostridium species [21] (Table 1). Many studies have shown that diets rich in non-digestible carbohydrates steadily increase the number of bifidobacteria and lactobacilli [22]. For example, a diet rich in whole wheat or wheat bran is associated with an increase in bifidobacteria and lactobacilli [23]. Other non-digestible carbohydrates, such as resistant starch and whole-wheat barley, also appear to increase the number of intestinal microbiota such as Rumenococci, E. rectale, and Roseburia spp. [24,25].

It is well-known that the risk of cardiovascular disease is closely related to the daily lipid intake. Several studies have shown that a diet high in saturated fat increases the number of anaerobic microbiota and Bacteroides spp.[26]. The number of Clostridium organisms also increases in the presence of a high saturated fat diet, but the number of lactobacilli decreases [27]. In contrast, an unsaturated fat diet results in an increase in the number of Bifidobacterium in the feces compared to baseline, while also leading to a decrease in fasting blood glucose [28].

Protein intake is divided into plant proteins and anima proteins. A large number of studies have shown that protein consumption is positively correlated with the overall microbial diversity. Excessive intake of dairy products can cause an increase in Bacteroides and Clostridium spp., while the number of bifidobacteria is relatively reduced [29,30]. It has been reported that the intake of whey and pea protein extracts can increase the number of bifidobacteria and lactobacilli in the intestine, while whey can also reduce the pathogenic Bacteroides fragilis and Clostridium perfringens [31].

Therefore, the daily diet determines the physiological state and abundance of the intestinal microbiota. Thus, clinicians can provide dietary guidance for patients with gastrointestinal cancer that will help reduce their disease burden or may improve the response to treatment.

\section{Nutritional Support for Patients with Gastrointestinal Tumors}

Patients with gastrointestinal tumors often develop malnutrition during the perioperative period. The causes of malnutrition are two-fold, involving both inadequate intake of nutrients and increased consumption of nutrients and energy. Tumor-induced pain or localized compression can lead to eating or emptying disorders. Inadequate intake can lead to cachexia and early satiety, which stimulates the hypothalamus to produce factors that further worsen anorexia [32]. In addition, malnutrition in cancer patients often leads to complications such as nausea, vomiting, and coagulopathy [33]. In patients with cancer, the local cellular metabolism is hyperactive, with increased glucose consumption as a result of the tumor-associated Warburg effect (anaerobic glycolysis) [34]. The host's proteins are also consumed to increase the formation of the tumor cells' proteins, resulting in a negative nitrogen balance in the body.

Bacterial infections can easily lead to microbial disorders in patients with gastrointestinal tumors, reducing postoperative recovery and the tolerance of radiotherapy and chemotherapy [35]. Immune function is impaired by malnutrition, which leads to abnormal lymphocyte development, decreased macrophage function, and the abnormal secretion of cytokines [36]. Damage to the immune function leads to an increase in infection and a decrease in the patients' prognosis [37]. Immune dysfunction also promotes tumorigenesis. For example, tumor-associated macrophages stimulate the proliferation of cancer cells and the epithelial-mesenchymal transition by accelerating the formation of blood vessels and lymphatic vessels, promoting the metastasis of tumor cells [38]. Neutrophil elastic hydrolase also promotes tumor cell invasion [39]. Of note, T cells play a dual role in tumorigenesis and metastasis. In the early stages of tumorigenesis, T cells can control tumor progression [40]. Due to the sustained selective pressure of the effector response, tumor variants are selected and evade immune recognition. The immune system is then unable to eradicate these selected tumors, which can proliferate and metastasize [41].

Clinicians have found that malnutrition is common in patients with gastrointestinal cancer. In most patients with severe malnutrition, immediate surgery is not the best option. There is also currently no clear way to significantly change an individual's body composition in a short period of time before surgery. However, perioperative nutritional support can significantly improve the postoperative outcomes of patients with severe malnutrition. When early intestinal nutrition does not meet the requirements to alleviate malnutrition, adding microecological agents and immune nutrition has been advocated [42]. Nutritional support is especially important after surgery. Postoperative malnutrition in patients with cancer is associated with increased infection rates, decreased immune function, increased postoperative complications, and a reduced tolerance of radiotherapy and chemotherapy [33]. This not only increases the financial burden on patients, but also increases their risk of mortality. In order to more directly regulate post-operative inflammation and other complications, the implementation of intestinal microecological immunotherapy can provide not only energy and nitrogen, but also a replacement population of the microbiota normally in the human body [43]. This concept is expected to open up a new field of research.

\section{A New Weapon against Cancer: the Emergence of Micro-ecological Immunotherapy}

The intestinal microbes have rapidly become a focus of extensive research in a variety of fields. The gut microbiota 
is considered to be the hidden immune organ of the human body, regulating the function and homeostasis of many organs throughout the body [44]. Nutritional support plays a crucial role in the immune and postoperative recovery of cancer patients. Intestinal microbes are being used by researchers as a new type of ecological nutrient for the treatment of tumors [45]. Intestinal micro-ecological immunotherapy integrates intestinal immune nutrition with intestinal ecological nutrition. Micro-ecological preparations are added to strengthen the clinical efficacy of nutritional support, reduce the occurrence of complications, and exert therapeutic effects either directly or by increasing the tolerance to conventional therapies [46]. Special nutrients such as glutamine and arginine are added to traditional intestinal nutrition to stimulate the body to produce an immune response, reduce excessive inflammation, and protect the integrity of the intestinal mucosa [47]. In a study of the effects of glutamine-based nutritional support on the perioperative period of patients with advanced gastric cancer, Wang $\mathrm{J}$ et al. found that the supplement protected the intestinal mucosal barrier function, reduced the adverse reaction rate and increased the patient quality of life [48].

Omega-3 polyunsaturated fatty acids have anti-inflammatory effects and are also used to reduce postoperative infections and complications [49]. Xie $\mathrm{H}$ et al. found that perioperative intestinal nutritional support is superior to preoperative and postoperative nutritional support in pre- venting postoperative infections and complications. Moreover, Wilhelm SM et al. used a combination of arginine and omega-3 polyunsaturated fatty acids to effectively reduce infection rates and improve patient survival of colorectal cancer $[49,50]$.

While intestinal nutritional support enhances the immunity of cancer patients and improves the prognosis of patients, the emergence of microbial preparations means that intestinal ecological nutrition can also be provided. Microbiota, especially probiotics, can be incorporated in the patient's daily nutrition, where the microbes will regulate the intestinal micro-ecological environment and reconstitutes the bacterial homeostasis, preventing the invasion of pathogenic bacteria [51]. The innovation of intestinal ecological immune nutrition lies in the combination of immune nutrition and microbial preparations (Figure 1). In support of this concept, Tan CK et al. gave patients microbial preparations before surgery, and found that this significantly promoted the recovery of postoperative intestinal function in patients with colorectal cancer and shortened their hospital stay [52]. Microbiota can also stimulate toll-like receptors (TLRs), trigger complex molecular cascades, produce cytokines and activate $\mathrm{T}$ cells [53]. Studies have shown that the regeneration of $T$ cells can also be induced by metabolites produced by the microbiota.

Based on the current understanding, several major bacterial metabolites play key roles in the pathogenesis of

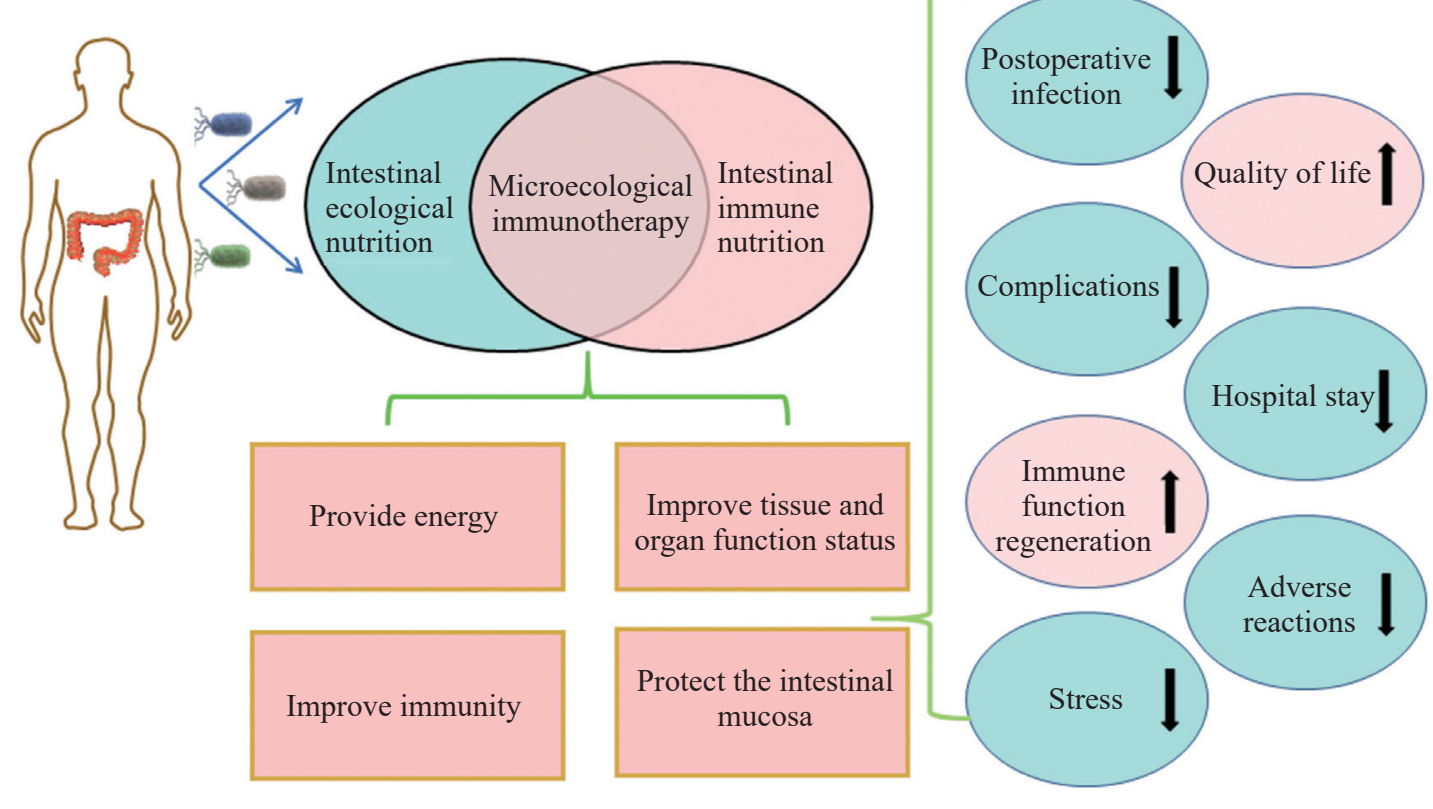

Figure 1 Introduction to micro-ecological immunotherapy. Micro-ecological immunotherapy is a new type of nutritional therapy that combines intestinal ecological nutrition with intestinal immune nutrition. It is based on the immunological factors included in intestinal immune nutrition, such as arginine, glutamic acid and omega-3 polyunsaturated fatty acids. This is combined with microbial preparations, such as probiotics, to provide nutrition and anti-tumor effects. Microecological immunotherapy provides energy, improves the tissue and organ functional status, improves immunity, protects the intestinal mucosa, and maintains the intestinal microbiota. Micro-ecological immunotherapy can reduce the risk of postoperative infections, reduce the rates of complications and adverse reactions, and promote the regeneration of immune cells, so that patients can improve their survival and quality of life and reduce their hospitalization time. 
colorectal cancer and the immune microenvironment that permits the growth of these cancers. These metabolites include SCFAs, polyamines, secondary bile acids, and phytochemicals. Their roles in colorectal cancer have been widely documented $[54,55]$. Several mechanisms have been proposed to underlie the effects of probiotics in the prevention and treatment of gastrointestinal tumors. First, some probiotics are capable of inducing anti-proliferative and apoptotic processes in colorectal cancer cells. For example, Propionibacterium freudenreichii induces apoptosis in colorectal and gastric cancers by producing SCFAs. This is because colorectal cancer cells can use the glycolysis pathway to create an acidic environment in the gastrointestinal tract, which results in the production of high SCFAs by Propionibacterium freudenreichii [53-56]. SCFAs block the proliferation of colorectal cancer cells primarily in the G1 phase of cell division [57]. Bifidobacteria can also inhibit the growth of colorectal cancer cells by stopping the cell cycle and increasing alkaline phosphatase activity during G0/G1. Alkaline phosphatase is associated with a reduced degree of malignancy in cancer cells [58,59]. This finding suggests that the addition of specific probiotic bacteria to lactic acid products can provide patients with both good postoperative nutrition and some therapeutic benefits. Microbes have also been shown to exert apoptosis via multiple mechanisms in HT29 and HCT116 cell lines; exerting cytotoxic effects on cancer cells, secreting specific cytotoxic metabolites, and positively regulating the $\mathrm{C}$-fos gene [60].

Second, probiotics can enhance the function of the intestinal epithelial barrier. SCFAs can activate adenosine monophosphate-activated protein kinase to enhance the tight junctions of the intestinal epithelium and form a strong barrier. This protein kinase is a key factor that regulates the energy metabolism of human colon cells. In vitro and in vivo experiments have demonstrated that supplementation of SCFAs enhances this intestinal barrier [54].

Third, probiotics can inhibit the enzymatic activity of pathogenic bacteria. The intestinal purification of toxins and absorption of nutrients largely relies on enzymes produced by various microbiota. Disordered microbiota was result in the release of a large number of oncogenic enzymes, such as $\beta$-glucuronidase, $\beta$-glucosidase and nitroreductase $[61,62]$. These enzymes are susceptible to toxic carcinogenic metabolites such as hydrogen sulfide, acetaldehyde aromatic amines, and secondary cholates, which can inhabit these products [63]. Therefore, probiotics can reduce the production of carcinogens by reducing the activity of these enzymes. For example, lactic acid bacteria reduce the dehydroxylation of primary bile acids [64]. The combination of various probiotics and vegetables can reduce the level of carcinogenic products such as $\beta$-glucuronidase, which are increased by the consumption of protein and fat. Probiotics can also participate in regulating the immune function. Studies have shown that probiotics interact with TLRs to produce anti-inflammatory cytokines [65]. Certain types of lactic acid bacteria increase the activity of regulatory $\mathrm{T}$ cells (Tregs), enhance the antibacterial phagocytic activity of peripheral blood neutrophils, and increase the activity of natural killer cells [66]. Soluble substances produced by probiotics may also induce a systemic immune response through secondary gastrointestinal antigen-presenting cells.

We have recently explored the roles of PD-1 and PDL1 in regulating the effects of microbial metabolites, with a focus on colorectal cancer and its microenvironment. Tumor immunotherapy is currently a hot topic of research. The PD-1 receptor is an inhibitory receptor expressed by activated $\mathrm{T}$ lymphocytes, $\mathrm{B}$ cells, natural killer $\mathrm{T}$ cells (NKT) and Treg cells, making it an important target for immunotherapy. PD-1 plays a key role in regulating T cell function in peripheral tissues, where it recognizes PD-L1 and PD-L2. These two ligands are expressed not only on antigen-presenting cells (APCs), but also on the surface of lymphoid tissues and non-lymphoid tissues (including tumor cells). PD-1 contains an immunoreceptor tyrosine-based inhibition motif (ITIM) and an immunoreceptor tyrosinestimulated motif (ITSM). When PD-1 recognizes its ligand, this interaction induces the phosphorylation of tyrosine residues in the ITSM, leads to the recruitment of tyrosine phosphatase SHP2, induces Zap70 dephosphorylation and inactivation in T cells, and down-regulates TCR signaling [67]. Therefore, PD-1 down-regulates the activity of T cells and inhibits the normal immune response. When PD-1 binds to PD-L1 in the tumor microenvironment, the function of $\mathrm{T}$ cells is impaired, allowing tumor cells to evade immune surveillance. Many studies have shown that blocking PD-1 or PD-L1 restores T cell function [68].

As noted above, micro-ecological immunotherapy is an excellent method for providing tumor control (Figure 2). Some intestinal microbiota can affect the interaction between PD-1 and PD-L1. For example, Sivan A et al. [71] compared melanoma cells from two different mice. These mice had different microbiota but were genetically similar. They found that bifidobacteria had a positive effect on activated APCs, which can effectively control tumors and IFN- $\gamma$ secretion [69]. The researchers also found that PD-1 expression is involved in experimental colitis. Wildtype (WT) mice had severe wasting disease caused by experimentally-induced colitis, whereas PD-1 deficient mice did not develop colonic inflammation [70]. Sivan A et al. compared the growth of mouse melanoma in mice with different commensal microbiota and observed differences in spontaneous anti-tumor immunity that were eliminated after co-administration or fecal metastasis [71]. Subsequent $16 \mathrm{~S}$ ribosomal RNA sequencing revealed that Bifidobacteria have anti-tumor effects. The effects of oral administration of Bifidobacteria alone on tumors were the same as those of PD-L1-specific antibody treatment, and combination therapy almost eliminated tumor growth [71]. When Wang $\mathrm{T}$ et al. administered Lactobacillus reuteri to 
regulate intestinal microbiota, it alleviated PD-1 antibodyinduced autoimmunity, thereby enabling immunotherapy to produce the desired immune response without significant immunopathology [72]. This suggests that the resistance of PD-1-deficient mice to colitis depends on their intestinal microbiota. These experiments provided new ideas about the potential mechanisms underlying micro-ecological immunotherapy. The addition of specific microbiota to micro-ecological immunotherapy can thus assist tumor immunotherapy, reduce complications, and provide greater opportunities for tumor elimination.

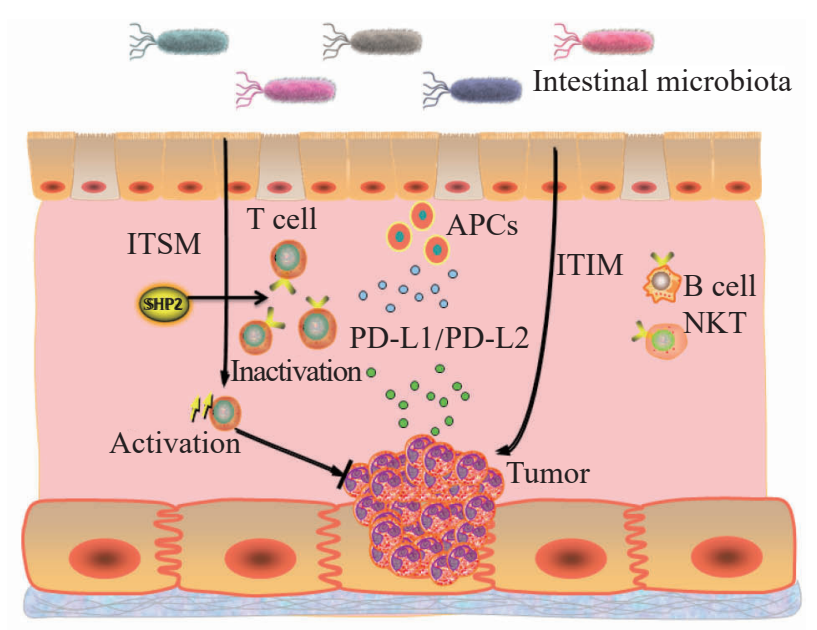

Figure 2 The intestinal microbiota is involved in regulating the relationship between PD-1, PD-L1 and tumorigenesis. PD-1 is produced by a variety of cells, including $\mathrm{T}$ cells, $\mathrm{B}$ cells, and NK cells. It recognizes PD-L1 on the surface of tumor cells and APCs, and induces an immunoreceptor tyrosine stimulating motif (ITSM). Tyrosine is dephosphorylated and inactivated via the ITSM, inhibiting T cell activity. Microbiota may be involved in the process of immune recognition, preventing the binding of PD-1 to PD-L1, thereby inhibiting tumor growth and progression.

In the perioperative period, intestinal ecological immune nutrition can improve the intestinal barrier, thus effectively reducing the stress response following the operation. In the surgical treatment of enteropathy, the use of this nutritional method can increase the diversity of the intestinal microbiota and reduce the symptoms of the digestive tract. At present, intestinal eco-immune nutrition mainly involves the addition of probiotics for nutritional intervention [65]. Sharaf LK et al. found that the administration of a combination of probiotics and celecoxib during the week before the induction of colorectal cancer in mice decreased the expression of proto-oncogene K-ras and increased the expression of $p 53$ [73]. These results implied that the addition of probiotics seem to help activate a tumor suppressor gene, maintain the normal cell activity and cell cycle, and effectively control the development of colorectal cancer. The researchers subsequently treated 140 patients with colorectal cancer receiving chemotherapy with supplemental probiotics and intestinal nutrition, while the control group received a placebo for eight weeks. The results showed that the level of inflammatory markers related to the side effects of chemotherapy were reduced in the probiotic and intestinal nutrition group. Moreover, this reduction was more significant than that associated with traditional therapies [74].

Intestinal ecological immune nutrition shows obvious advantages in the promotion of postoperative recovery. In a study by Consoli ML et al., oral probiotics reduced the time to the first major complication, the duration of the first infection and the time to the first bowel movement [75]. Intestinal immune nutrition improves the patient's survival and discharge rate, as well as the quality of life. The interaction of probiotics with immature dendritic cells results in activation of the complement cascade and increased proliferation of NK cells [76]. The combination of various probiotics also promotes the apoptosis of colonic cells with DNA damage caused by carcinogens, downregulates oncogenic enzymes, and enhances immune regulation [77].

In summary, the main mechanisms underlying the effects of micro-ecological immunotherapy in the intestine are the provision of energy, improvement of the functional state of tissues and organs, improvement of the immune function, protection of the intestinal mucosa and maintenance of the balance of intestinal microbiota.

\section{Evaluation and Future Prospects of Micro-ecological Immunotherapy}

The development of intestinal immune nutrition has been rapid. Researchers have discovered a powerful anticancer probiotic with ancient origins: Kefir [78]. Bioactive peptides in kefir induce macrophage activation and phagocytosis [79]. The active peptides also induce ROSmediated apoptosis and activate $\mathrm{Ca}^{2+} / \mathrm{Mg}^{2+}$-dependent endonuclease for DNA cleavage [80]. The intake of kefir reduces TGF- $\alpha$ and Bcl2 by increasing the secretion of Bax and inducing apoptosis in cancer cell [78]. Kefir has antitumor, anti-proliferative and apoptosis-inducing effects in vitro and in vivo, and can be used for the treatment of colorectal cancer, breast cancer and lung cancer [79].

The intestinal microbiota are not only useful in the treatment or prevention of tumors, but can also be used for tumor screening. By using sequencing technology to comprehensively analyze the types and quantities of intestinal microbes in a population, clinicians can successfully distinguish between colorectal adenomas, cancers, and non-colorectal adenomas [81]. This may be related to an increase in the abundance of proteobacteria in the stool of patients with colorectal adenomas and cancers. Moreover, it has recently been found that intestinal microbial-based predictive models are more accurate in the diagnosis of colorectal cancer than standard fecal occult blood tests [82]. This suggests that changes in the microbiota in the feces can be used as a non-invasive 
sensitive screening indicator for detecting the health status of high-risk populations, permitting the early diagnosis of colorectal cancer, thereby permitting the early treatment of colorectal cancer, reducing mortality and prolonging the patient's life-span. By analyzing the shared functions between different microbiota, we can further clarify the microbial markers of gastrointestinal tumors. Establishing a causal relationship between microbiota and cancer is expected to help lock in which microbes are associated with various effects, making it possible to alter the genes of the microbes, and prevent and inhibit tumor growth associated with the microbes and their environment.

\section{Conflict of Interests}

There is no conflict of interest to declare.

\section{Funding}

This work was supported by grants from the Training Project of Key Talents of Youth Medicine in Jiangsu Province, China [No. QNRC2016330], the Academic Science and Technology Innovation Fund for College Students [No. X20180714], and the Yangzhou Science and Technology Bureau Social Development Project [No. YZ2018087].

\section{References}

1. Dieterich W, Schink M, Zopf Y. Microbiota in the gastrointestinal tract. Med Sci (Basel) 2018;6(4). pii: E116. doi: 10.3390/medsci6040116.

2. Joukar F, Mavaddati S, Mansour-Ghanaei F, Samadani AA. Gut microbiota as a positive potential therapeutic factor in carcinogenesis: an overview of microbiota-targeted therapy. J Gastrointest Cancer 2019; doi: 10.1007/s12029-019-00237-6.

3. Peng L, Wu LG, Li B, Zhao J, Wen LM. Early enteral nutrition improves intestinal immune barrier in a rat model of severe acute pancreatitis. J Hepatobiliary Pancreat Sci 2016;23(11):681-7.

4. Wang QH. Effect of ecological immune-enhanced enteral nutrition on patients with gastrointestinal fistulas. Eur Rev Med Pharmacol Sci 2017;21(9):2261-7.

5. Wang J, Tian S, Yu H, Wang J, Zhu W. Response of colonic mucosa-associated microbiota composition, mucosal immune homeostasis, and barrier function to early life galactooligosaccharides intervention in suckling piglets. J Agric Food Chem 2019;67(2):578-8.

6. Zhang S, Wang Q, Zhou C, Chen K, Chang H, Xiao W, Gao Y. Colorectal cancer, radiotherapy and gut microbiota. Chin J Cancer Res 2019;31(1):212-2.

7. Gu X, Zheng R, Xia C, Zeng H, Zhang S, Zou X, Yang Z, Li H, Chen W. Interactions between life expectancy and the incidence and mortality rates of cancer in China: a population-based cluster analysis. Cancer Commun (Lond) 2018;38(1):44.

8. Zhang P, Deng R, Liu K, Shuai XM, Bai J, Chang WL, Gao JB, Cai KL, Wang GB, Tao KX. Clinicopathologic features and prognosis of primary gastrointestinal stromal tumor patients under 35 years of age: A 10-year retrospective study. J Surg Oncol 2016;114(8):977-81.
9. Kountouras J, Doulberis M, Papaefthymiou A, Polyzos SA, Touloumtzi M, Vardaka E, Kapetanakis N, Liatsos C, Gavalas E, Anastasiadis S, Tzivras D, Katsinelos P. The relationship between Helicobacter pylori-related microbiota dysbiosis and gastrointestinal tract pathologies. Scand J Gastroenterol 2019;54(6):806-7.

10. Jeyamani L, Jayarajan J, Leelakrishnan V, Swaminathan M. CagA and VacA genes of Helicobacter pylori and their clinical relevance. Indian J Pathol Microbiol 2018;61(1):66-9.

11. Suzuki M, Mimuro H, Suzuki T, Park M, Yamamoto T, Sasakawa C. Interaction of CagA with Crk plays an important role in Helicobacter pylori-induced loss of gastric epithelial cell adhesion. J Exp Med. 2005;202(9):1235-47.

12. Huang X, Wang C, Sun J, Luo J, You J, Liao L, Li M. Clinical value of CagA, c-Met, PI3K and Beclin-1 expressed in gastric cancer and their association with prognosis. Oncol Lett 2018;15(1):947-55.

13. Ricci V. Relationship between VacA toxin and host cell autophagy in helicobacter pylori infection of the human stomach: a few answers, many questions. Toxins (Basel) 2016;8(7): pii: E203. doi: 10.3390/toxins 8070203 .

14. Ladeira MS, Bueno RC, Dos Santos BF, Pinto CL, Prado RP, Silveira MG, Rodrigues MA, Bartchewsky W, Jr., Pedrazzoli J, Jr., Ribeiro ML, Salvadori DM. Relationship among oxidative DNA damage, gastric mucosal density and the relevance of cagA, vacA and iceA genotypes of Helicobacter pylori. Dig Dis Sci 2008;53(1):248-55.

15. de Almeida CV, Taddei A, Amedei A. The controversial role of Enterococcus faecalis in colorectal cancer. Therap Adv Gastroenterol 2018;11:1756284818783606.

16. Sarshar M, Scribano D, Marazzato M, Ambrosi C, Aprea MR, Aleandri M, Pronio A, Longhi C, Nicoletti M, Zagaglia C, Palamara AT, Conte MP. Genetic diversity, phylogroup distribution and virulence gene profile of pks positive Escherichia coli colonizing human intestinal polyps. Microb Pathog 2017;112:274-8.

17. Dai Z, Zhang J, Wu Q, Chen J, Liu J, Wang L, Chen C, Xu J, Zhang H, Shi C, Li Z, Fang H, Lin C, Tang D, Wang D. The role of microbiota in the development of colorectal cancer. Int J Cancer 2019;145(8):203241.

18. Scotto d'Abusco AS, Sanfilippo L, Menozzi MG, Pantosti A. Activity and role of BFT, an enterotoxin produced by Bacteroides fragilis. J Nat Toxins 2000;9(3):267-80.

19. Chen Y, Peng Y, Yu J, Chen T, Wu Y, Shi L, Li Q, Wu J, Fu X. Invasive Fusobacterium nucleatum activates beta-catenin signaling in colorectal cancer via a TLR4/P-PAK1 cascade. Oncotarget 2017;8(19):31802-14.

20. Eid N, Enani S, Walton G, Corona G, Costabile A, Gibson G, Rowland I, Spencer JP. The impact of date palm fruits and their component polyphenols, on gut microbial ecology, bacterial metabolites and colon cancer cell proliferation. J Nutr Sci 2014;3:e46.

21. Francavilla R, Calasso M, Calace L, Siragusa S, Ndagijimana M, Vernocchi P, Brunetti L, Mancino G, Tedeschi G, Guerzoni E, Indrio F, Laghi L, Miniello VL, Gobbetti M, De Angelis M. Effect of lactose on gut microbiota and metabolome of infants with cow's milk allergy. Pediatr Allergy Immunol 2012;23(5):420-7.

22. Keim NL, Martin RJ. Dietary whole grain-microbiota interactions: insights into mechanisms for human health. Adv Nutr 2014;5(5):556-7. 
23. Leitch EC, Walker AW, Duncan SH, Holtrop G, Flint HJ. Selective colonization of insoluble substrates by human faecal bacteria. Environ Microbiol 2007;9(3):667-79.

24. Liu Z, Lin X, Huang G, Zhang W, Rao P, Ni L. Prebiotic effects of almonds and almond skins on intestinal microbiota in healthy adult humans. Anaerobe 2014;26:1-6.

25. Yu ZT, Liu B, Mukherjee P, Newburg DS. Trametes versicolor extract modifies human fecal microbiota composition in vitro. Plant Foods Hum Nutr 2013;68(2):107-12.

26. Wu GD, Chen J, Hoffmann C, Bittinger K, Chen YY, Keilbaugh SA, Bewtra M, Knights D, Walters WA, Knight R, Sinha R, Gilroy E, Gupta K, Baldassano R, Nessel L, Li H, Bushman FD, Lewis JD. Linking long-term dietary patterns with gut microbial enterotypes. Science 2011;334(6052):105-8.

27. Fava F, Gitau R, Griffin BA, Gibson GR, Tuohy KM, Lovegrove JA. The type and quantity of dietary fat and carbohydrate alter faecal microbiome and short-chain fatty acid excretion in a metabolic syndrome 'at-risk' population. Int J Obes (Lond) 2013;37(2):216-23.

28. Urwin HJ, Miles EA, Noakes PS, Kremmyda LS, Vlachava M, Diaper ND, Godfrey KM, Calder PC, Vulevic J, Yaqoob P. Effect of salmon consumption during pregnancy on maternal and infant faecal microbiota, secretory IgA and calprotectin. Br J Nutr 2014;111(5):77384.

29. Jantchou P, Morois S, Clavel-Chapelon F, Boutron-Ruault MC, Carbonnel F. Animal protein intake and risk of inflammatory bowel disease: The E3N prospective study. Am J Gastroenterol 2010;105(10):2195-201

30. Eeckhaut V, Machiels K, Perrier C, Romero C, Maes S, Flahou B, Steppe M, Haesebrouck F, Sas B, Ducatelle R, Vermeire S, Van Immerseel F. Butyricicoccus pullicaecorum in inflammatory bowel disease. Gut 2013;62(12):1745-52

31. Swiatecka D, Narbad A, Ridgway KP, Kostyra H. The study on the impact of glycated pea proteins on human intestinal bacteria. Int J Food Microbiol 2011;145(1):267-72.

32. Osumi H, Takahari D, Chin K, Ogura M, Ichimura T, Wakatsuki T, Suzuki T, Ota Y, Nakayama I, Ooki A, Suenaga M, Shinozaki E, Yamaguchi K. Modified FOLFOX6 as a first-line treatment for patients with advanced gastric cancer with massive ascites or inadequate oral intake. Onco Targets Ther 2018;11:8301-7.

33. Kufeldt J, Viehrig M, Schweikert D, Fritsche A, Bamberg M, Adolph M. Treatment of malnutrition decreases complication rates and shortens the length of hospital stays in a radiation oncology department. Strahlenther Onkol 2018;194(11):1049-59.

34. Tekade RK, Sun X. The Warburg effect and glucose-derived cancer theranostics. Drug Discov Today 2017;22(11):1637-53.

35. Yue M. Bacterial persistent infection at the interface between host and microbiota. Clin Infect Dis 2016;62(10):1325-6.

36. Rytter MJ, Kolte L, Briend A, Friis H, Christensen VB. The immune system in children with malnutrition--a systematic review. PLoS One 2014;9(8):e105017

37. Gavalas NG, Karadimou A, Dimopoulos MA, Bamias A. Immune response in ovarian cancer: how is the immune system involved in prognosis and therapy: potential for treatment utilization. Clin Dev Immunol 2010;2010:791603.
38. Chen JY, He LI, Zhang HX, Sun MM, Chen KS. Effects of tumor-associated macrophages on the proliferation and migration of esophageal cancer-associated lymphatic endothelial cells. J Biol Regul Homeost Agents 2018;32(2):207-18.

39. Jiang T, Qiao M, Zhao C, Li X, Gao G, Su C, Ren S, Zhou C. Pretreatment neutrophil-to- lymphocyte ratio is associated with outcome of advanced-stage cancer patients treated with immunotherapy: a meta-analysis. Cancer Immunol Immunother 2018;67(5):713-27.

40. Li W, Ma H, Zhang J, Zhu L, Wang C, Yang Y. Unraveling the roles of CD44/CD24 and ALDH1 as cancer stem cell markers in tumorigenesis and metastasis. Sci Rep 2017;7(1):13856.

41. Malmberg KJ, Sohlberg E, Goodridge JP, Ljunggren HG. Immune selection during tumor checkpoint inhibition therapy paves way for NK-cell “missing self” recognition. Immunogenetics 2017;69(8-9):54756.

42. West MA, Wischmeyer PE, Grocott MPW. Prehabilitation and nutritional support to improve perioperative outcomes. Curr Anesthesiol Rep 2017;7(4):340-9.

43. Wang MQ, Li Y, Sun CH. [The relationship between gut microbiota and diet and nutrition related diseases]. Zhonghua Yu Fang Yi Xue Za Zhi 2018;52(2):195-200.

44. Vivarelli S, Salemi R, Candido S, Falzone L, Santagati M, Stefani S, Torino F, Banna GL, Tonini G, Libra M. Gut microbiota and cancer: from pathogenesis to therapy. Cancers (Basel) 2019;11(1).pii: E38. doi: 10.3390/cancers 11010038 .

45. Wu X, Zhang T, Chen X, Ji G, Zhang F. Microbiota transplantation: Targeting cancer treatment. Cancer lett 2019;452:144-51.

46. Staffas A, Burgos da Silva M, Slingerland AE, Lazrak A, Bare CJ, Holman CD, Docampo MD, Shono Y, Durham B, Pickard AJ, Cross JR, Stein-Thoeringer C, Velardi E, Tsai JJ, Jahn L, Jay H, Lieberman S, Smith OM, Pamer EG, Peled JU, Cohen DE, Jenq RR, van den Brink MRM. Nutritional support from the intestinal microbiota improves hematopoietic reconstitution after bone marrow transplantation in mice. Cell Host Microbe 2018;23(4):447-57.e4.

47. Yavas C, Yavas G, Acar H, Toy H, Yuce D, Akyurek S, Ata O. Amelioration of radiation-induced acute inflammation and mucosal atrophy by beta-hydroxy-beta-methylbutyrate, L-glutamine, and L-arginine: results of an experimental study. Support Care Cancer 2013;21(3):883-8.

48. Wang J, Li Y, Qi Y. Effect of glutamine-enriched nutritional support on intestinal mucosal barrier function, MMP-2, MMP-9 and immune function in patients with advanced gastric cancer during perioperative chemotherapy. Oncol Lett 2017;14(3):3606-10.

49. Wilhelm SM, Kale-Pradhan PB. Combination of arginine and omega-3 fatty acids enteral nutrition in critically ill and surgical patients: a meta-analysis. Expert Rev Clin Pharmacol 2010;3(4):459-69.

50. Xie H, Chang YN. Omega-3 polyunsaturated fatty acids in the prevention of postoperative complications in colorectal cancer: a meta-analysis. Onco Targets Ther 2016;9:7435-43.

51. Lin L, Zhang J. Role of intestinal microbiota and metabolites on gut homeostasis and human diseases. BMC Immunol 2017;18(1):2.

52. Tan CK, Said S, Rajandram R, Wang Z, Roslani AC, Chin KF. Pre-surgical administration of microbial cell preparation in colorectal cancer patients: a randomized controlled trial. World J Surg 2016;40(8):1985-92. 
53. Mukherji A, Kobiita A, Ye T, Chambon P. Homeostasis in intestinal epithelium is orchestrated by the circadian clock and microbiota cues transduced by TLRs. Cell 2013;153(4):812-27.

54. Spiljar M, Merkler D, Trajkovski M. The immune system bridges the gut microbiota with systemic energy homeostasis: focus on TLRs, mucosal barrier, and SCFAs. Front Immunol 2017;8:1353.

55. Thanissery R, Winston JA, Theriot CM. Inhibition of spore germination, growth, and toxin activity of clinically relevant $\mathrm{C}$. difficile strains by gut microbiota derived secondary bile acids. Anaerobe 2017;45:86-100.

56. Eslami M, Yousefi B, Kokhaei P, Hemati M, Nejad ZR, Arabkari V, Namdar A. Importance of probiotics in the prevention and treatment of colorectal cancer. J Cell Physiol 2019;234(10):17127-43.

57. Wang G, Yu Y, Wang YZ, Wang JJ, Guan R, Sun Y, Shi F, Gao J, $\mathrm{Fu}$ XL. Role of SCFAs in gut microbiome and glycolysis for colorectal cancer therapy. J Cell Physiol 2019;234(10):17023-49.

58. Coakley M, Johnson MC, McGrath E, Rahman S, Ross RP, Fitzgerald GF, Devery R, Stanton C. Intestinal bifidobacteria that produce trans-9, trans-11 conjugated linoleic acid: a fatty acid with antiproliferative activity against human colon SW480 and HT-29 cancer cells. Nutr Cancer 2006;56(1):95-102.

59. Hung HY, Chen JS, Chien Y, Tang R, Hsieh PS, Wen S, You YT, You JF, Chiang JM. Preoperative alkaline phosphatase elevation was associated with poor survival in colorectal cancer patients. Int $\mathrm{J}$ Colorectal Dis 2017;32(12):1775-8

60. Sharma M, Shukla G. Metabiotics: one step ahead of probiotics; an insight into mechanisms involved in anticancerous effect in colorectal cancer. Front Microbiol. 2016;7:1940.

61. Yang W, Wei B, Yan R. Amoxapine demonstrates incomplete inhibition of beta-glucuronidase activity from human gut microbiota. SLAS Discov 2018;23(1):76-83.

62. McCormack E, Silden E, West RM, Pavlin T, Micklem DR, Lorens JB, Haug BE, Cooper ME, Gjertsen BT. Nitroreductase, a near-infrared reporter platform for in vivo time-domain optical imaging of metastatic cancer. Cancer Res 2013;73(4):1276-86.

63. Cao X, Ding L, Xie ZZ, Yang Y, Whiteman M, Moore PK, Bian JS A review of hydrogen sulfide synthesis, metabolism, and measurement: is modulation of hydrogen sulfide a novel therapeutic for cancer? Antioxid Redox Signal 2019;31(1):1-38.

64. Del Carmen S, de Moreno de LeBlanc A, Levit R, Azevedo V, Langella P, Bermudez-Humaran LG, LeBlanc JG. Anti-cancer effect of lactic acid bacteria expressing antioxidant enzymes or IL-10 in a colorectal cancer mouse model. Int Immunopharmacol 2017;42:122-9

65. Xie H, Lu Q, Wang H, Zhu X, Guan Z. Effects of probiotics combined with enteral nutrition on immune function and inflammatory response in postoperative patients with gastric cancer. J Buon 2018;23(3):678-83

66. Zhu D, Chen X, Wu J, Ju Y, Feng J, Lu G, Ouyang M, Ren B, Li Y. [Effect of perioperative intestinal probiotics on intestinal flora and immune function in patients with colorectal cancer]. Nan Fang Yi Ke Da Xue Xue Bao 2012;32(8):1190-3.

67. Botticelli A, Zizzari I, Mazzuca F, Ascierto PA, Putignani L, Marchetti L, Napoletano C, Nuti M, Marchetti P. Cross-talk between microbiota and immune fitness to steer and control response to anti PD-1/
PDL-1 treatment. Oncotarget 2017;8(5):8890-9.

68. Patel J, Crawford JM. Microbiota-regulated outcomes of human cancer immunotherapy via the PD-1/PD-L1 axis. Biochemistry 2018;57(6):901-3.

69. Eri T, Kawahata K, Kanzaki T, Imamura M, Michishita K, Akahira L, Bannai E, Yoshikawa N, Kimura Y, Satoh T, Uematsu S, Tanaka H, Yamamoto K. Intestinal microbiota link lymphopenia to murine autoimmunity via PD-1(+)CXCR5(-/dim) B-helper T cell induction. Sci Rep 2017;7:46037.

70. Park SJ, Kim JH, Song MY, Sung YC, Lee SW, Park Y. PD-1 deficiency protects experimental colitis via alteration of gut microbiota. BMB Rep 2017;50(11):578-83.

71. Sivan A, Corrales L, Hubert N, Williams JB, Aquino-Michaels K, Earley ZM, Benyamin FW, Lei YM, Jabri B, Alegre ML, Chang EB, Gajewski TF. Commensal Bifidobacterium promotes antitumor immunity and facilitates anti-PD-L1 efficacy. Science 2015;350(6264):10849.

72. Wang T, Zheng N, Luo Q, Jiang L, He B, Yuan X, Shen L. Probiotics Lactobacillus reuteri abrogates immune checkpoint blockade-associated colitis by inhibiting group 3 innate lymphoid cells. Front Immunol 2019;10:1235.

73. Sharaf LK, Sharma M, Chandel D, Shukla G. Prophylactic intervention of probiotics (L.acidophilus, L.rhamnosus GG) and celecoxib modulate Bax-mediated apoptosis in 1,2-dimethylhydrazine-induced experimental colon carcinogenesis. BMC Cancer 2018;18(1):1111.

74. Golkhalkhali B, Rajandram R, Paliany AS, Ho GF, Wan Ishak WZ, Johari CS, Chin KF. Strain-specific probiotic (microbial cell preparation) and omega-3 fatty acid in modulating quality of life and inflammatory markers in colorectal cancer patients: a randomized controlled trial. Asia Pac J Clin Oncol 2018;14(3):179-91.

75. Consoli ML, da Silva RS, Nicoli JR, Bruna-Romero O, da Silva RG, de Vasconcelos Generoso S, Correia MI. Randomized clinical trial: impact of oral administration of saccharomyces boulardii on gene expression of intestinal cytokines in patients undergoing colon resection. JPEN J Parenter Enteral Nutr 2016;40(8):1114-21.

76. Esmaeili SA, Mahmoudi M, Rezaieyazdi Z, Sahebari M, Tabasi N, Sahebkar A, Rastin M. Generation of tolerogenic dendritic cells using Lactobacillus rhamnosus and Lactobacillus delbrueckii as tolerogenic probiotics. J Cell Biochem 2018;119(9):7865-72.

77. Burns AJ, Rowland IR. Antigenotoxicity of probiotics and prebiotics on faecal water-induced DNA damage in human colon adenocarcinoma cells. Mutat Res 2004;551(1-2):233-43.

78. Sharifi M, Moridnia A, Mortazavi D, Salehi M, Bagheri M, Sheikhi A. Kefir: a powerful probiotics with anticancer properties. Med Oncol 2017;34(11):183.

79. Rafie N, Golpour Hamedani S, Ghiasvand R, Miraghajani M. Kefir and Cancer: A systematic review of literatures. Arch Iran Med 2015;18(12):852-7.

80. Zamberi NR, Abu N, Mohamed NE, Nordin N, Keong YS, Beh BK, Zakaria ZA, Nik Abdul Rahman NM, Alitheen NB. The antimetastatic and antiangiogenesis effects of kefir water on murine breast cancer cells. Integr Cancer Ther 2016;15(4):NP53-66.

81. Mailhe M, Ricaboni D, Vitton V, Gonzalez JM, Bachar D, Dubourg G, Cadoret F, Robert C, Delerce J, Levasseur A, Fournier PE, Ange- 
lakis E, Lagier JC, Raoult D. Repertoire of the gut microbiota from stomach to colon using culturomics and next-generation sequencing. BMC Microbiol 2018;18(1):157.

82. Yachida S, Mizutani S, Shiroma H, Shiba S, Nakajima T, Sakamoto T, Watanabe H, Masuda K, Nishimoto Y, Kubo M, Hosoda F, Rokutan H, Matsumoto M, Takamaru H, Yamada M, Matsuda T, Iwasaki M, Ya- maji T, Yachida T, Soga T, Kurokawa K, Toyoda A, Ogura Y, Hayashi T, Hatakeyama M, Nakagama H, Saito Y, Fukuda S, Shibata T, Yamada T. Metagenomic and metabolomic analyses reveal distinct stage-specific phenotypes of the gut microbiota in colorectal cancer. Nat Med 2019;25(6):968-76. 